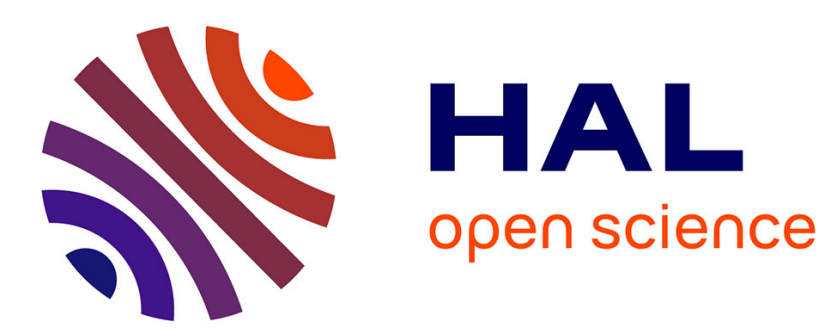

\title{
Publisher's Announcements and Piracy-Monitoring Devices in Software Adoption
}

\author{
Eric Darmon, Alexandra Rufini, Dominique Torre
}

\section{To cite this version:}

Eric Darmon, Alexandra Rufini, Dominique Torre. Publisher's Announcements and Piracy-Monitoring Devices in Software Adoption. Annals of Economics and Statistics, 2014, 115-116, pp.409-430. halshs00929792

\section{HAL Id: halshs-00929792 \\ https://shs.hal.science/halshs-00929792}

Submitted on 16 Jan 2014

HAL is a multi-disciplinary open access archive for the deposit and dissemination of scientific research documents, whether they are published or not. The documents may come from teaching and research institutions in France or abroad, or from public or private research centers.
L'archive ouverte pluridisciplinaire HAL, est destinée au dépôt et à la diffusion de documents scientifiques de niveau recherche, publiés ou non, émanant des établissements d'enseignement et de recherche français ou étrangers, des laboratoires publics ou privés. 


\title{
Publishers' announcements and piracy-monitoring devices in software adoption
}

\author{
Eric DARMON*
}

\author{
Alexandra RUFINI ${ }^{\dagger}$
}

Dominique TORRE ${ }^{\dagger}$

\author{
October 29, 2013
}

\begin{abstract}
Résumé
Ce papier analyse la stratégie d'un éditeur de logiciel vis-à-vis du piratage. Dans un contexte d'adoption caractérisée par une incertitude sur la qualité (bien d'expérience) et des coûts de piratage hétérogènes, un éditeur peut choisir le prix et la qualité du logiciel distribué ainsi qu'un niveau d'effort ou de surveillance des utilisateurs. Les utilisateurs peuvent choisir de pirater ou d'acheter le logiciel de l'éditeur durant l'une des deux périodes considérées ou de ne pas l'adopter. Entre les deux périodes, un mécanisme de bouche-à-oreille permet de révéler imparfaitement la qualité du logiciel. Dans ce contexte, nous montrons sous quelles conditions une politique de tolérance vis-à-vis du piratage peut être profitable pour l'éditeur. Nous envisageons aussi la possibilité pour un éditeur d'annoncer des niveaux de prix et de surveillance futurs qu'il n'appliquerait pas parfaitement durant la deuxième période d'adoption. Une telle politique, faussement permissive en matière de piratage, s'avère encore plus efficace. Néanmoins, seule la politique permissive vis-à-vis du piratage demeure robuste dès lors qu'on augmente le niveau de sophistication des anticipations des utilisateurs quant à l'appréhension de la stratégie de l'éditeur.
\end{abstract}

Classification JEL : D43, D83, K42, L15

Mots-clés : stratégie de distribution d'un logiciel, piratage, bien d'expérience, fausses annonces

\begin{abstract}
In this paper, we investigate the distribution strategy of a software publisher. The user adoption context is characterized by uncertainty about quality (experience good)and heterogeneous piracy costs. Users can purchase or get unauthorized/illegal copies (digital piracy) of the software during two periods (or not adopt at all). Between these two periods, users can acquire information through word-of-mouth. To maximize profit, the publisher needs to decide about price, quality and level of monitoring of piracy. We show that the software publisher can profit from accommodation a certain level of piracy of the product. We add to the literature by explicitly considering the opportunity for the publisher to cheat about future price and monitoring levels (misleading announcements). This strategy that is falsely permissive towards piracy, can sometimes appear more profitable. However, when the degree of sophistication of user expectations about the publisher's strategy increases, only a strategy that is permissive (with respect to piracy) with non misleading announcements remains robust.
\end{abstract}

JEL Codes: D43, D83, K42, L15

Keywords: software distribution strategy, piracy, experience good, misleading announcements

${ }^{*}$ Université de Rennes 1 - CREM - CNRS, 7 place Hoche, 35065 Rennes Cedex, France. E-mail: eric.darmon@univ-rennes1.fr

†Université Nice Sophia-Antipolis - GREDEG - CNRS, 250 rue Albert Einstein, 06560 Valbonne, France. E-mail: alexandra.rufini@gredeg.cnrs.fr, dominique.torre@gredeg.cnrs.fr 


\section{Introduction}

Since the early 2000, the massive adoption of information and communication technologies (ICT) by firms and households has had a major impact on the production and distribution strategies of producers of digital goods (software, cultural goods, media/newspaper, etc.). Because of the intrinsic characteristics of such goods (non rivalry, lower distribution costs, increasing returns to scale), this massive adoption has increased the opportunities and the risks for digital good producers (see Shapiro and Varian [1998] for a seminal analysis). In particular, these risks seem to be magnified as reproduction costs diminish (or disappear) and as the degree of vertical differentiation between the original good and the copy is very low. Although concerns about piracy existed even before the diffusion of ICT(see e.g. Conner and Rumelt [1991] for a seminal theoretical analysis of copying and Varian [2005] for a review of this literature), their use (because of the relative easiness to use of downloading platforms such as e.g. peer-to-peer networks) has often been considered a facilitator for distributing digital goods (whether legally or illegally). Thus, the negative effects on sales and then profit are often pointed out in these industries.

However, file-sharing networks and online communities also generate new opportunities for profit. Thanks to the diffusion of ICT, increasing the connectivity between users has become much cheaper, which, in turn, induces new network effects or reinforces existing ones. Digital goods producers can then profitably exploit the existence of such externalities (e.g. increase in users' willingness to pay, opportunities to lock-in users) that overall impact positively on their profits. There is a growing literature that investigates these effects and studies how piracy and profit may be reconciled depending on the type of good - mainly software, music and video (see Belleflamme and Peitz [2010]) - and on the demand characteristics (see Peitz and Waelbroeck [2006a]). This article focuses on the case of software. Software typically display several types of network externalities: compatibility-driven externalities either direct or indirect (i.e. compatibility with a complementary software that generates indirect revenues), and especially information driven externalities notably (see Peitz and Waelbroeck [2006a] for a complete description of all potential externalities).

Here we focus on informational externalities. As well as being non-material, software is also most frequently an experience good (Nelson [1970]). For that reason, users may need additional information to evaluate the benefits associated with software use. This additional knowledge about software quality in turn may foster software adoption. For that reason, software publishers often use freeware or shareware distribution strategies. Both are based on versioning through the distribution of limited versions (with respect to time or functionalities) to help to reveal to users the exact quality of the software (see Haruvy and Prasad [2005] for a survey). The tacit assumption behind such strategies is that an individual test (also described as direct sampling) can decrease individual learning costs and reduce the information asymmetry in product quality. Although monitoring the distribution of a unauthorized good is more difficult, a "permissive" strategy towards piracy could have the same effect on individual learning. ${ }^{1}$.

\footnotetext{
${ }^{1}$ cf. e.g. Stolpe [2000] for an early empirical analysis showing that some software publishers lower their efforts about software protection if the magnitude of software externalities is perceived to be high.
} 
Our paper builds on the literature on sampling ${ }^{2}$. Several theoretical papers study the effect of direct sampling in the case of a monopolistic market (cf. Peitz and Waelbroeck [2006b], Gopal et Bhattacharjee [2006], Duchêne and Waelbroeck [2005]). However, as Takeyama [2009]notes, these contributions often do not investigate the precise effect of another type of sampling (indirect sampling). Because ICT use decreases the costs of communication among users, information flows between potential users of a software are intensified which increases the scale of the sampling effect. Thus, Takeyama [2009] proposes a model that integrates an indirect sampling effect and shows that the strategic introduction of unauthorized copies through piracy, in some cases can reduce information asymmetry. Also, her model assumes that an illegal copy has the same marginal cost for each user and that original and illegal software are vertically differentiated. Besides, the distribution between pirates and legal consumers is either governed by an exogenous parameter or perfectly monitored by the software publisher. Our model builds on this literature but relaxes some of these restrictive assumptions. In particular, we fully endogenize the tradeoff between piracy (unauthorized software use), legal purchase and non adoption. We assume also that potential software users are heterogeneous vis-à-vis the cost associated with piracy. This may be grounded either on heterogeneous technical skills and/or on different moral attitudes towards illegal activities such as illegal downloading. In our model, the software publisher has the opportunity to select an effort level to monitor piracy (e.g. using more or less restrictive protecting devices). This monitoring policy is publicly known and influence users' attitudes to piracy. However, when the monitoring policy is not fully enforced, the choice of whether to get unauthorized copies or not is endogenous. We also consider a two-stage model to capture the idea that the software publisher is able to revise its price and piracy-monitoring strategies. This two-step model also enables us to consider the timing of adoption: to our knowledge, this possibility is not considered in the piracy literature although is an issue in the software industry. We include the opportunity for the software publisher to make strategic announcements about its future price and piracy monitoring strategies.

In this setting, we show that a software publisher may have an incentive to implement a permissive strategy regarding piracy behaviours i.e. to tolerate a moderate level of piracy of its software during the pre-adoption (i.e. first) stage. We show that this first strategy contributes to information disclosure and increases users' willingness to pay for the legal product. We show also that a second strategy may be available to the software publisher. This consists in making an announcement about a future permissiveness towards piracy. However, this announcement is misleading i.e. the publisher deliberately cheats about the future levels of price and monitoring: the levels implemented will be different from those announced to users. This second strategy may be even more beneficial for the software publisher than the permissive strategy. In this case, legal buyers disclose information during the pre-adoption stage and those users who planned to get unauthorized copies of the software later are eventually induced to buy it at the higher

\footnotetext{
${ }^{2}$ As Liebowitz [2005] points out, the sampling effect was originally referred to as the 'exposure effect' in the literature and consists in experiencing a good (that is downloading in the context of a digital good) before adoption. Hence, sampling is a way for users to check that the experienced item matches their tastes and preferences. Although sampling was first designed for music/video items and thought at an individual scale (i.e. a user tests and potentially adopts the item), this notion has thus be extended to account for all types of digital (experience) goods and word-of-mouth diffusion (i.e. a user tests and disclose information to another user) thus generating network effects.
} 
price. Since the publisher's announcements are not implemented, the strategy based on cheating no longer holds in a context of more sophisticated user expectations and the first type of strategy is robust to increases in the sophistication of user expectations.

Section 2 presents the Model. Section 3 presents the potential adoption equilibrium outcomes and strategies. Section 4 discusses the results and concludes.

\section{The model}

The model focuses on the behaviour and the strategic decisions of a software publisher that aims to launch a new software in the market. We consider software as an experience good, so its characteristics are only imperfectly observable. In that context, potential users have to decide whether or not to adopt the new software proposed by the software publisher. If they decide to adopt, they need also to decide about the right time for adoption and how to acquire the software (buying legally or getting unauthorized copies of the software).

\subsection{The software publisher}

We consider a two-period model. The publisher determines the price $p_{t}$ of the software at each time period $t(t=\{1,2\})$ and its quality level $q, q \in[0, \bar{q}]$. Below the quality level $b(0<b<\bar{q})$, the software provides basic functionalities only. When this quality level is reached, the software is endowed with advanced functionalities, which are unobservable before use.

The production cost of the software, denoted $C(q)$, is positively related to its quality level. For simplicity, ${ }^{3}$ we suppose that $C(q)=\underline{C}$ when $q \in[0, b]$, and $C(q)=\bar{C},(\underline{C}<\bar{C})$, when $q \in] b, \bar{q}]$.

The publisher is able indirectly to influence the level of piracy (unauthorized use) of its software by enforcing some monitoring of piracy behaviours. The intensity of this monitoring is measured by $\lambda_{t}\left(\lambda_{t} \in[0,1]\right)$. For each time period $t=\{1,2\}$, this monitoring level $\lambda_{t}$ corresponds to the probability of a user of being caught using unauthorized copies of the software. The lowest monitoring level $\left(\lambda_{t}=0\right)$ corresponds to a zero probability of being caught ; the highest monitoring level $\left(\lambda_{t}=1\right)$ means that being caught is a certain event for pirate users. This monitoring is assumed to be costless for the publisher. This assumption ensures that potential piracy permissiveness is not caused by the cost of monitoring supported by the software publisher.

In our model, the software publisher publicly announces, at time 1 , its price policy and her attitude toward piracy for the two periods. Hence, the publisher announces the levels of price $p_{1}$ and monitoring $\lambda_{1}$ at time 1 , but also the level of price $p_{2}^{a}$ and the monitoring level $\lambda_{2}^{a}$ which could be applied at time 2 .

\subsection{Potential users}

There are $m$ potential software users uniformly distributed in the segment $[\underline{c}, \bar{c}]$. We denote $c_{i},(i=1,2 \ldots m)$, the piracy cost of the potential user $i$. This cost can vary from

\footnotetext{
${ }^{3}$ This assumption enables us to treat quality as a discrete binary variable. This considerably simplifies the mathematical treatment of the model without qualitative incidences on the propositions stated here.
} 
one user to another given technical factors (heterogeneous technical ability to get unauthorized copies of the software), psychological factors (different morale attitudes toward unauthorized activities and their potential sanctions) or cultural factors (different social environments which approve/disapprove more or less of piracy behaviours).

Each potential user, supposed risk-neutral, could adopt the software at time 1 or at time 2, by either buying or getting unauthorized copies of it (piracy). Once acquired, the software can be further without any additional charge. If some users do not adopt the new software (further "reservation strategy"), they draw the utility level $\bar{b}$, associated with the use of an old generation of software. At time 1, users imperfectly estimate the characteristics of the software. They expect the utility associated with the quality of the software $q$ at the level $q^{e}$.

To avoid trivial individual choices, we formulate two assumptions.

Assumption 1. If the new software provides only basic functionalities, the old software provides a level of utility at least as high as provided by the new software, such that $b \leq \bar{b}$.

This assumption eliminates the case where only the basic functionalities would ensure the adoption of the software, making the adoption problem trivial. Also, given that advanced functionalities are not observable, the expected quality level $q^{e}$ has to be bounded. If the users' expectations are too pessimistic (i.e. such that $q^{e}<\bar{b}$ ) the reservation strategy always dominates other strategies and no user would ever adopt. Conversely, if they are too optimistic $\left(q^{e}>\bar{q}\right)$ delayed adoption (i.e. adoption at time 2$)$ would never occur. Hence, potential users' expectations cannot be higher than the maximum quality set by the publisher. Assumption 2 preserves the possibility of early and/or delayed adoption.

Assumption 2. The maximum quality level of the new software expected by users is bounded by the maximum quality level set by the publisher and provides a higher level of utility than the utility level provided by the older-generation software, such that $\bar{b} \leq q^{e} \leq \bar{q}$.

Let $m_{1}^{u}$ denote the number of early adopters, i.e. the users who adopt the software at time 1 , with $0 \leq m_{1}^{u} \leq 1$. Once early users have adopted, information about the quality of the software diffuses to potential users through a word-of-mouth process. This defines an informational externality that we suppose is proportional to the number of early adopters. This information disclosure leads other potential users to correct their estimation of quality by an amount of $k\left(m_{1}^{u} / m\right)\left(q-q^{e}\right)$, (where $k$ measures the magnitude of the information diffusion $(0 \leq k \leq 1)$.

At each time, potential users take into account all the available information (price, monitoring level, software quality) to make their current decision (reservation, buying the software or getting unauthorized copies of it) in order to maximize their intertemporal utility. Here we consider basic expectations: users do not anticipate that the software publisher will lie in announcing future price levels and monitoring levels. The case of more sophisticated types of expectations is discussed in Section 4.

\subsection{The structure of the sequential game}

- At time 0 , the software publisher determines the level of the control variables $\left\{p_{1}, p_{2}, p_{2}^{a}, \lambda_{1}, \lambda_{2}, \lambda_{2}^{a}, q\right\}$ that maximizes its non-discounted intertemporal profit. ${ }^{4}$

\footnotetext{
${ }^{4}$ We could have considered a discount factor on profit. However, introducing this parameter would not change results qualitatively (types of equilibrium strategies and outcomes).
} 
The publisher announces publicly levels of price and monitoring $\left\{p_{1}, p_{2}^{a}, \lambda_{1}, \lambda_{2}^{a}\right\}$.

- At time 1, potential users make their current decisions (reservation, buying the software or getting unauthorized copies of it) maximizing their intertemporal utility (sum of the instantaneous non-actualized utility levels), given the available information on the control variables $\left\{p_{1}, p_{2}^{a}, \lambda_{1}, \lambda_{2}^{a}\right\}$ and their current estimations about software quality $q^{e}$. Adoption decisions are enforced immediately.

- A time 2, the software publisher implements the monitoring level $\lambda_{2}$ and proposes the price $p_{2}$. The levels of these control variables were chosen at time 0 but could be different from those previously announced. Potential users that did not adopt during time 1 revise their decisions given the available information about software quality and given the price and the monitoring levels actually implemented by the publisher.

Announcements are said to be 'non misleading' if $p_{2}^{a}=p_{2}$ and $\lambda_{2}^{a}=\lambda_{2}$, and misleading otherwise. In the latter case, and because of the structure of the game (i.e. no new piece of information or no state of nature intervene between the two adoption periods for the software publisher), the software publisher's strategy would be based on deliberate cheating since the publisher would know at the beginning of the game that it would never implement at time 2 the announcements made at time 1 .

The intertemporal non-actualized profit of the software publisher is defined by (1):

$$
\begin{aligned}
\pi\left(p_{1}, p_{2}, p_{2}^{a}, \lambda_{1}, \lambda_{2}, \lambda_{2}^{a}, q\right)= & m_{1}^{s}\left(p_{1}, p_{2}^{a}, \lambda_{1}, \lambda_{2}^{a}, q\right) p_{1} \\
& +m_{2}^{s}\left(p_{2}, \lambda_{2}, q\right) p_{2}-C(q)
\end{aligned}
$$

where $m_{1}^{s}\left(p_{1}, p_{2}^{a}, \lambda_{1}, \lambda_{2}^{a}, q\right)$ and $m_{2}^{s}\left(p_{2}, \lambda_{2}, q\right)$ are the number of pieces of software sold at time 1 and time 2 respectively.

The timing of adoption defines a sequential game where the publisher plays first and integrates the optimal intertemporal responses of potential users in its decisions. In so doing, the publisher includes the revision process of users' expectations and decisions. The resulting values of the control variables for both publisher and potential users are depicted by the Stackelberg equilibrium and we consider the perfect sub-game Nash equilibria.

\section{Results}

First, we study the choices of users given the piracy costs. From that, we deduce the potential distributions of users among the possible strategies (buying, piracy, reservation): each defines an 'outcome' of the game. Second, we select, among all the potential outcomes of the game, those that match the publisher's optimal decisions and focus on the cases where piracy is (rationally) accommodated by the software publisher.

\subsection{Users' strategies and outcomes of the game}

At each time, potential users determine, revise or confirm their strategies, given the levels of the observed and announced variables and given their piracy cost. 
For a given set of the control variables, the outcomes of the game are determined according to the adoption time structure. First, potential users choose at time 1 to adopt or not and how to to adopt (buying the software or getting unauthorized copies of it). Second, those who do not adopt the software at time 1, choose again at time 2 among the different actions (buying, getting unauthorized copies of the software, reservation). Because potential users have heterogeneous piracy costs, they may not always choose the same strategy at a given time period.

\subsubsection{Possible outcomes of the game}

Given Assumptions 1 and 2, the software publisher initially chooses a quality level $q$, $(0 \leq q \leq \bar{q})$, and potential users can select one of the five strategies at time $1(1.1,1.2$, 1.3, 1.4 and 1.5) depicted by Figure 1. Note that each strategy is associated with a payoff depending on the parameters $\bar{b}, q^{e}, \underline{c}, \bar{c}$ and on the announced values of the control variables $\left\{p_{1}, p_{2}^{a}, \lambda_{1}, \lambda_{2}^{a}\right\}$.

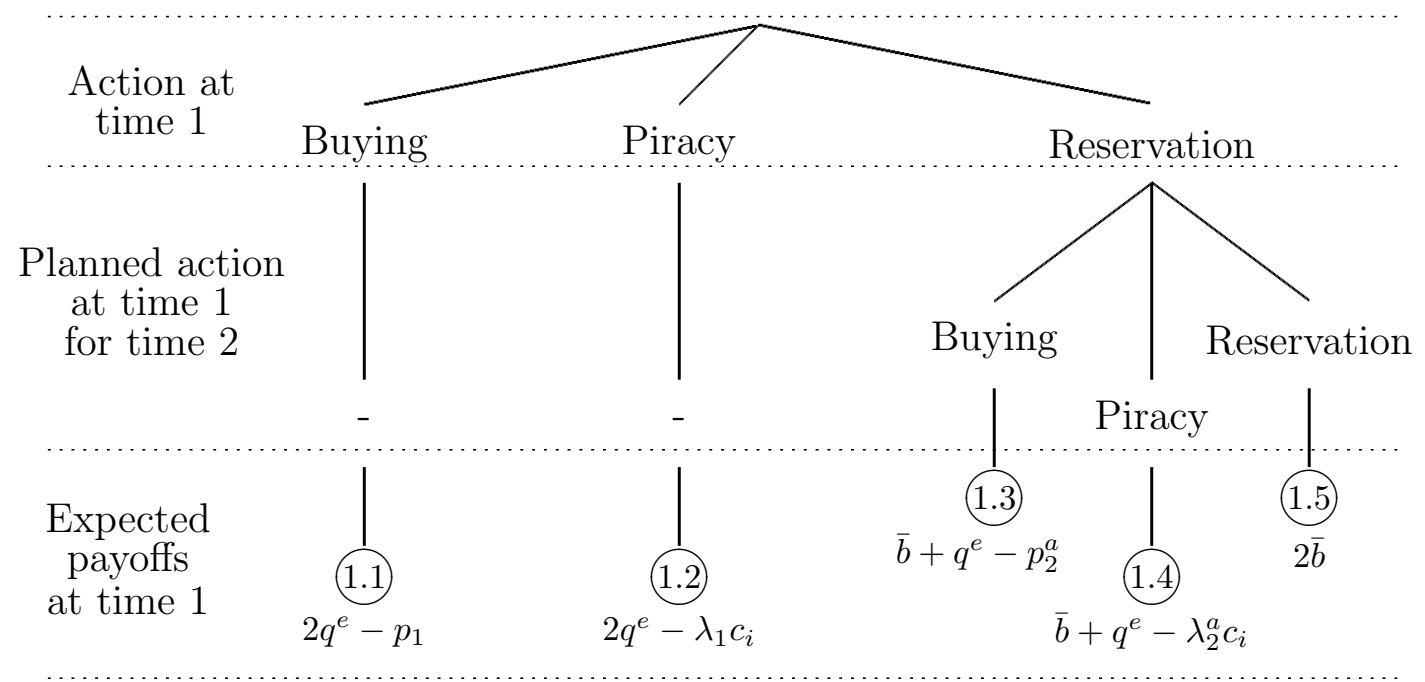

Figure 1: User $i$ 's decision tree at time 1

At time 2, the users' estimation of the quality of the software may change. Early adopters (buyers and/or hackers) may decide not to adopt the software if its quality is not associated with a level of utility greater than $\bar{b}$. In this case, information diffuses to those users who have not yet adopted and the expected quality of the software is damaged. However, this possible re-use of the previous software by early adopters does not impact on their actions (buying or piracy) since those actions are forgone. Nor does it impact directly on the publisher's profit (no possible refund). However, note that it may impact on this profit indirectly since unfavourable word-of-mouth information may drive users' expectations down.

For that reason, we concentrate on the decisions of those potential users who did not adopt at time 1. Those who chose Strategies 1.3, 1.4 or 1.5 may, at time 2, confirm or revise their previous choice. At time 2, the same three options are available to them: buying the software, getting unauthorized copies of it or definitively not adopting. The choice of those users is depicted in Figure 2. 


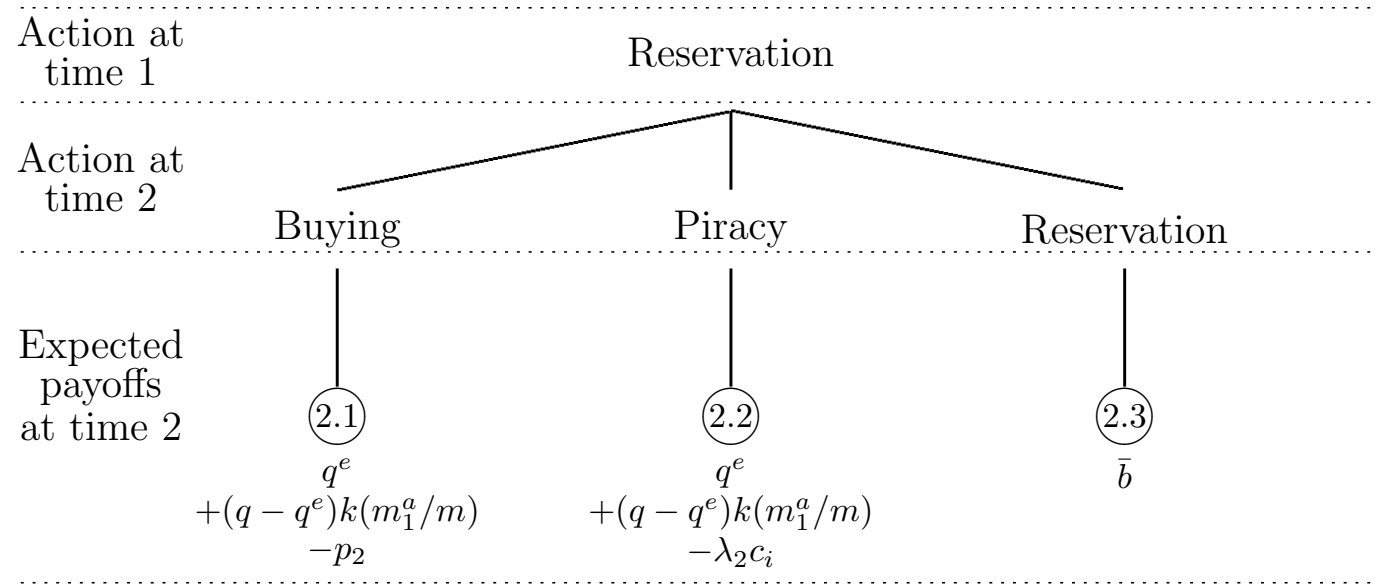

Figure 2: User's $i$ decision tree at time 2

(choice for those users that chose the reservation strategy at time 1)

In the limit case where two payoffs are equal (i.e. when two strategies yield exactly the same level of utility), we assume that potential users always prefer legal purchase to piracy or reservation, on the one hand, and piracy to reservation on the other hand. Comparison of users' payoffs as defined in Figures 1 and 2 leads to Lemma 1:

Lemma 1. At time 1, users cannot distribute among Strategy 1.1 (buying at time 1) and 1.3 (reservation at time 1 and expected purchase at time 2), or among 1.1 and 1.5 (reservation at time 1 and expected reservation at time 2), or among 1.3 and 1.5. At time 2, users who previously chose the reservation strategy cannot distribute among Strategy 2.1 (reservation at time 1 and buying at time 2) and 2.3 (reservation at time 1 and 2).

Proof: Since users differ by their piracy $\operatorname{cost} c_{i}$ and not by their willingness to pay, the payoff associated with Strategy 1.1 is greater than or equal to (resp. less than or equal to) the payoff associated with Strategy 1.3 whatever $i(i=1,2 \ldots m)$. That is, potential users all prefer either 1.1 to 1.3 or vice versa but all users make the same decision. The same deductions apply to the comparison of the payoffs associated with 1.1 vs $1.5,1.3$ vs $1.5,2.1$ vs 2.3

\subsubsection{Outcomes of the game matching the publisher's optimal decisions}

Lemma 1 helps to reduce the number of possible outcomes of the game. However, the number of these outcomes can still be high. On the software publisher's side, it is obviously not optimal to choose some values of the control variables $\left\{q, p_{1}, p_{2}^{a}, p_{2}, \lambda_{1}, \lambda_{2}^{a}, \lambda_{2}\right\}$ such that all potential users would adopt the software at time 1 or 2 by getting unauthorized copies of it only. Thus, and because the publisher plays first, we can consider only those strategies compatible with optimal actions on the publisher's side, which significantly reduces the final number of candidate outcomes.

As a consequence, a distribution of users such that all of them would choose Strategy 1.2 (early piracy) is excluded since it would result in a zero profit. In the same way, at time 2, the publisher should ensure that at least one part of those who did not adopt, will buy the software. Therefore, a situation where all potential users would choose Strategy 2.2 (delayed piracy) and/or 2.3 (reservation) is never optimal for the publisher. We set 
aside these outcomes.

Also, our objective in this paper is to identify the conditions for a distribution strategy based on piracy to be profitable. For that, we need to ensure that a strategy that would deter piracy completely is at least feasible and compatible with users' incentives. If the publisher is able to deter piracy totally but does not do so, this means that it is in its interest to tolerate piracy. This leads to Assumption 3 which excludes the case where some users might find it optimal to get unauthorized copies while at the same time the publisher enforces the maximum monitoring level. ${ }^{5}$.

Assumption 3. Enforcing the maximum monitoring level $\left(\lambda_{t}=1, t=\{1,2\}\right)$ discourages the user endowed with the lowest piracy cost from getting unauthorized copies of the software at time $t$.

Given Assumption 3 and the expected payoffs associated with each user's strategy, we can formulate Lemmas 2 to 5 .

Lemma 2. When some sales occur at time 1, the publisher will never choose $\lambda_{1}<1$.

Proof: The publisher chooses the control variables $\left\{p_{1}, p_{2}^{a}, \lambda_{1}, \lambda_{2}^{a}\right\}$ such that at least one fraction of all potential users chooses Strategy 1.1. Given Lemma 1, potential users distribute among Strategies 1.1 and/or 1.2 and/or 1.4. If the publisher chooses $\lambda_{1}=1$, Strategy 1.2. is dominated (cf. Assumption 3). Decreasing $\lambda_{1}$ below its maximum level $\lambda_{1}=1$ leads either to a decrease in sales at time 1 (because part of the potential users may choose Strategy 1.2 rather than 1.1) or to a decrease in sales at time 2 (because part of the potential users may choose 1.2 rather than 1.4, meaning fewer users choosing 2.1 at time 2)

Corollary 1. When some sales occur at time 1, users cannot distribute among Strategy 1.1 (early buying) and Strategy 1.2 (early piracy).

The publisher may induce some users to choose Strategy 1.2 in order to diffuse information about software quality. This word-of-mouth process should provide incentives for reluctant users to buy the software at the next time period. However, when some sales are made at time 1 , it is always more profitable for the publisher to diffuse information through buyers rather than to do it through unauthorized users, given that users never buy the software once it has been previously illegally acquired.

Lemma 3. The publisher never chooses $\lambda_{2}<1$.

Proof: If some sales are made at time 1 for the control variables $\left\{p_{1}, p_{2}^{a}, \lambda_{1}, \lambda_{2}^{a}\right\}$, all the values of $\lambda_{2}$ ensure this equilibrium, and in particular $\lambda_{2}=1$. If some sales are made at time 2 , any decrease in $\lambda_{2}$ below its maximum level $\lambda_{2}=1$ may affect sales. Given the payoffs associated with the strategies 2.1 and 2.2 , the effect on sales, whatever is $p_{2}$, is a decrease in sales at time 2: decreasing $\lambda_{2}$ therefore, is inefficient for the publisher's side

\footnotetext{
${ }^{5}$ This inverse situation is investigated in Darmon et al. [2009] in a close but simplified setting in which the timing of adoption is the same but in which there is no opportunity for the software publisher to change its announced strategy between the two rounds. One key result of the present paper (Proposition 1) would be preserved. That means that Assumption 3 is not necessary for our result to hold. However, this working hypothesis considerably simplifies the presentation of the model to enable a complete treatment of strategic announcements.
} 
Corollary 2. The publisher always chooses its control variables $\left\{p_{1}, p_{2}^{a}, \lambda_{1}, \lambda_{2}^{a}\right\}$ such that no potential user selects Strategy 2.2.

These Lemmas and their corollaries indicate that the publisher should implement a permissive strategy towards piracy only at time 1, only if there are no sales at time 1.

Lemma 4. The publisher never announces the control variables $\left\{p_{1}, p_{2}^{a}, \lambda_{1}, \lambda_{2}^{a}\right\}$ such that no adoption will take place at time 1.

Proof: let us compare the profit associated with the values of the variables such that all potential users will adopt the software at time 2 with no early adoption, with the profit associated with the values of the control variables such that all potential users will adopt the software at time 1 . In both cases, the profit is written as $\pi_{t}=m p_{t}-\underline{C}$.

Consider first the case where the publisher announces the control variables $\left\{p_{1}, p_{2}^{a}, \lambda_{1}, \lambda_{2}^{a}\right\}$ such that no user adopts at time 1. Given Lemma 3, we know that the publisher will choose $\lambda_{2}=1$ and given Assumption 3, we deduce no potential user will choose Strategy 2.2. In this context, the publisher should choose $p_{2}$ as high as possible (note that the profit is linear in $p_{2}$ ) but such that users still prefer 2.1 rather than 2.3, i.e. : $p_{2} \leq q^{e}-\bar{b}$ which is associated with the following profit : $\pi^{\chi}=m\left(q^{e}-\bar{b}\right)-\underline{C}$.

Now consider the situation where the publisher announces a set of control variables $\left\{p_{1}, p_{2}^{a}, \lambda_{1}, \lambda_{2}^{a}\right\}$ such that all users will adopt the software at time 1 . In this case, the control variables $p_{2}^{a}, \lambda_{1}$ and $\lambda_{2}^{a}$ have to be sufficiently high.

Given Assumption 3, we know that $2\left(q^{e}-\bar{b}\right) \leq \underline{c}+q^{e}-\bar{b}$. This inequality ensures that if potential users prefer Strategy 1.1 to 1.5, they will also prefer Strategy 1.1 to 1.4. Since the profit is an increasing function of $p_{1}$, the optimal value of $p_{1}$ is defined by the more restrictive constraint, i.e. $p_{1}<2\left(q^{e}-\bar{b}\right)$. In this situation, the profit would be $\pi^{\psi}=2 m\left(q^{e}-\bar{b}\right)-\underline{C}$ in this situation.

Comparing the two profits reveals that $\pi^{\psi}>\pi^{\chi}$ whatever the values of the parameters, i.e. it is always more profitable to sell the software at time 1 rather than to sell it at time 2 if no potential users adopted at time 1

We can deduce from Lemma 4 that the publisher should always announce a set of control variables such that one part of potential users chooses Strategy 1.1 (early buying) or Strategy 1.2 (early piracy).

Lemma 5. The publisher never announces:

(i) a price $p_{2}^{a}$ inferior to $p_{1}$ or a monitoring level $\lambda_{2}^{a}$ inferior to $\lambda_{1}$ when sales are made at time 1 at equilibrium,

(ii) a monitoring level $\lambda_{2}^{a}$ inferior to $\lambda_{1}$ when sales are only made at time 2 at equilibrium.

Proof: (i) At equilibrium, if sales are made at time 1 at the price level $p_{1}$, announcing $p_{2}^{a}<p_{1}$ will have no effect if $p_{2}^{a}>p_{1}-\left(q^{e}-\bar{b}\right)$. Otherwise, if $p_{2}^{a}<p_{1}-\left(q^{e}-\bar{b}\right)$, then all potential users prefer Strategy 1.3 to Strategy 1.1, i.e. no potential user buys the software at time 1 , which is not compatible with the case studied here.

(ii) At equilibrium, if it is more profitable to sell at time 2 rather than to sell at time 1 , the publisher should accommodate some piracy at time 1 (cf. Lemma 4). The optimal level of piracy at time 1 can be determined thanks to the monitoring level $\lambda_{1}$. Announcing that $\lambda_{2}^{a}$ is less than $\lambda_{1}$ either may have no incidence or may restrict the level of piracy at time 1 (if the value of $\lambda_{2}^{a}$ is rather low). Any increase in $\lambda_{1}$ however has the same effect. 
Since any change in the optimal proportion of unauthorized users obtained by $\lambda_{2}^{a}$ can also be obtained by a change in the level of $\lambda_{1}$, it is not necessary at time 2 to announce a lower monitoring level than the one chosen at time 1

Corollary 3. It is not possible to have, simultaneously, some potential users choosing Strategy 1.2 (piracy at time 1) and some choosing Strategy 1.4 (reservation at time 1 and expectation of piracy at time 2).

Given Lemmas 1, 2, 3, 4 and 5 and their corollaries, only four outcomes of the game are possible (cf. Figure 3).

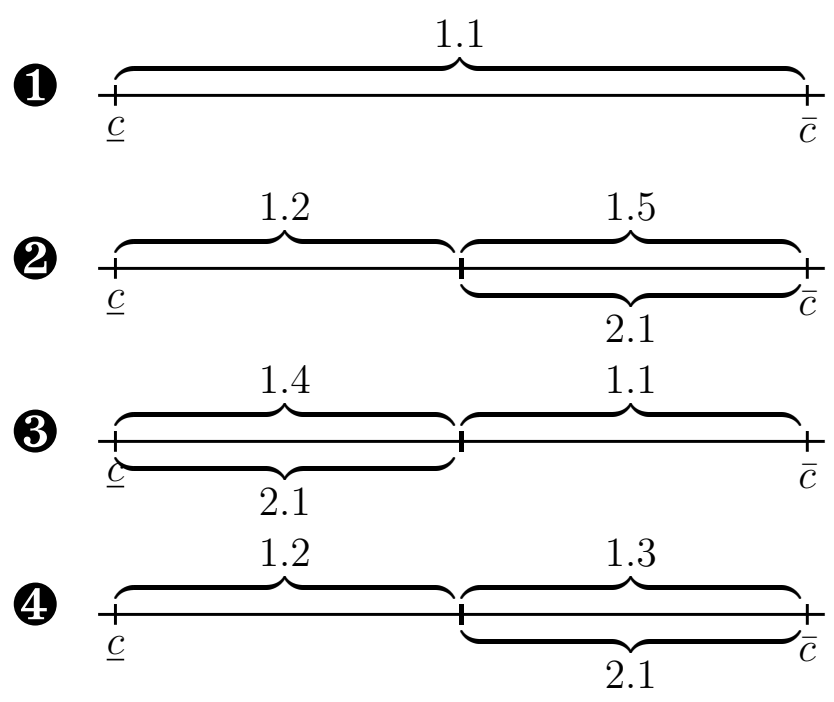

Figure 3: The four outcomes of the game

\subsection{The publisher's strategies}

As depicted in Figure 3, the four outcomes of the game fall into two categories. On the one hand, Outcomes 1 and 2 are characterized by non-misleading announcements (price and monitoring levels announced at time 0 are those implemented at time 2). However, Outcomes 1 and 2 differ in the level of quality $q$ chosen by the publisher. On the other hand, outcomes $(3$ and 4 are both based on misleading announcements. Note that these two outcomes correspond to the choice of a 'high' quality software $(q=\bar{q})$.

Hence, in the latter two cases, cheating is a part of the publisher's strategy. In Outcome 3, the publisher announces a monitoring level $\lambda_{2}^{a} \neq \lambda_{2}$ which it will not make effective at time 2 . The level of $\lambda_{2}^{a}$ announced at time 1 must be sufficiently low to motivate some users to choose Strategy 1.4. However, given Lemma 3, it is always optimal for the publisher to implement $\lambda_{2}=1$ at time 2, whatever her announcement at time 0 . In Outcome $\boldsymbol{4}$, the publisher announces a price $p_{2}^{a}$ lower than the price $p_{1}$, with the objective of motivating some users to postpone their purchase to time 2 instead of getting unauthorized copies of the software at time 1 (Strategy 1.3). In this case, the publisher has no interest in confirming such a low price at time 2 since the previous diffusion of favourable information about software quality enables the software publisher to sell it at a higher price. 
In the following sections, we study the publisher's strategies associated with each outcome of the game. For each case, we express the associated profit and determine the optimal strategy corresponding to this profit. We then separate the outcomes of the game associated with strategies based on non-misleading announcements (i.e., strategies such that the level of price and monitoring announced at time 0 is effective at time 2) from the outcomes associated with strategies based on misleading announcements.

We develop only those strategies associated with the game Outcomes (1), (2) et 3 . Outcome 4 is not analyzed in details here. Indeed, while Outcomes 2 and $\boldsymbol{4}$ correspond to different announcements made by the publisher, they correspond to exactly the same distribution of potential users among strategies. In each case, some users choose piracy during the early adoption stage while the others initially wait during that time period and end up buying the software at time 2 . In each case, the same proportion of users finally buys the software at time 2 , and at the same price. As a consequence, the publisher's profit is the same for the publisher for both outcomes. For reputation reasons, it seems realistic to suppose that the publisher might select a strategy based on cheating only if doing so allows it to earn an additional profit. Since this does not apply to the case here, Outcome 4 will never by considered as a relevant strategy by the publisher.

\subsubsection{Publisher's optimal strategy with non-misleading announcements}

Among the three possible publisher strategies, two are based on non-misleading announcements. For these two latter cases, the publisher announces at time 0 the price and the level of control that he will actually implement at time 2 . These outcomes correspond to opposite attitudes of the publisher towards piracy:

- Anti-piracy policy (labelled Strategy A): the publisher determines the optimal level of $\left\{p_{1}, p_{2}^{a}, p_{2}, \lambda_{1}, \lambda_{2}^{a}, \lambda_{2}, q\right\}$ such that all users will buy legally during time 1 ;

- Permissive policy (labelled Strategy B): the publisher determines the optimal level of $\left\{p_{1}, p_{2}^{a}, p_{2}, \lambda_{1}, \lambda_{2}^{a}, \lambda_{2}, q\right\}$ such that no user will buy during time 1 but some will do so at time 2 .

At Stackelberg equilibrium, the publisher's Strategy A is only compatible with the distribution of users associated with Outcome 1, while Strategy B is only compatible with the distribution of users associated with Outcome 2. We now consider the publisher's strategies $\mathrm{A}$ and $\mathrm{B}$ and the associated potential distribution of users.

Strategy A : In this case, the publisher determines the levels of its control variables so that all buyers buy at time 1 . However, potential users choose to actually buy the software only if Strategy 1.1 (early purchase) gives them a utility level greater than the utility they would obtain with another strategy, whatever their piracy $\operatorname{cost} c_{i}$. In this 
case, the utilities of users must satisfy the following conditions $(i \in[0, m])$ :

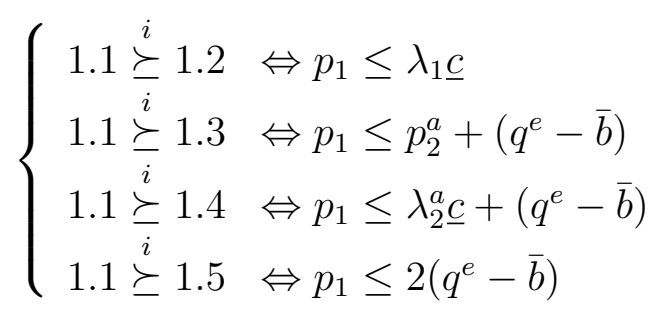

These four conditions jointly provide an upper bound for $p_{1}$. Given Assumptions 2 and 3, the first and third inequalities are satisfied as soon as the publisher announces and applies the maximum level of control, i.e. when $\lambda_{1}^{*}=1$ and $\lambda_{2}^{a *}=1$. As a consequence of Lemma 5, the publisher never chooses a price $p_{2}^{a}$ smaller than $p_{1}$ when the software is sold at time 2: the second inequality then is also verified when the publisher wants to sell at time 1. Given Assumption 2, there is a non-empty subset of positive values of $p_{1}$ satisfying the last inequality. Then, whatever the values of the utility parameters, there exists a compact and non-empty subset of values for $p_{1}$ that satisfy the four inequalities.

In order to determine the optimal level of the control variables compatible with this strategy, note that, for a price $p_{1}$ belonging to the compact set $\mathcal{C}$ defined by the four strategies and given Expression (1), the profit associated with the choice of Strategy A by the publisher can be expressed as follows: $\pi^{A}\left(q, p_{1}\right)=m p_{1}-C(q)$.

This expression always increases with $p_{1}$, and consequently its maximal value is obtained for the highest value of the price $p_{1}$ in the compact set $\mathcal{C}$. Given the definition of $C(q)$, the lowest possible quality level for the advanced functionalities is also optimal, given the impossibility to observe this quality at the time of full adoption. Since adoption ends up at time 1 , the actual price $p_{2}^{*}$ and the control levels $\lambda_{2}^{*}$ cannot be observed by users, and the optimal level of these variables are then indeterminate. Because of this, the publisher has no interest to cheat and to announce false levels of future price and monitoring levels (i.e. levels that will not be implemented in time 2). Assumption 3 excludes that the optimal price level $p_{1}$ could bind the constraints associated with $1.1 \succeq_{i}^{i} 1.2$ and $1.1 \succeq 1$. ${ }^{i}$. Similarly, Lemma 5 excludes that the constraint associated with $1.1 \succeq 1.3$ could be bounded. The constraint associated with $1.1 \underbrace{i} 1.5$ then is always bounded and the optimal solution has the form $\left\{q^{*}=b, p_{1}^{*}=p_{2}^{a *}=2\left(q^{e}-\bar{b}\right), \lambda_{1}^{*}=1, \lambda_{2}^{a *}=1, p_{2}^{*}, \lambda_{2}^{*}\right\}$, where $p_{2}^{*}$ and $\lambda_{2}^{*}$ take indeterminate values.

If the publisher selects Strategy A, it then chooses the maximum monitoring level. This choice increases the propensity of early adopters that prefer to buy rather than to get unauthorized copies. The publisher then chooses a minimal quality for the advanced functionalities of the software: since potential users have all adopted at time 1 , revelation of the low quality of the software at time 2 has no influence on the publisher's profit. Then the profit-maximizing quality level is also the one that minimizes the publisher's costs. Last, the publisher chooses for time 1 a price such that all users would immediately buy the software instead of getting unauthorized copies of the software. It is then sufficient that the publisher announces a sufficiently high price $p_{2}^{a}$ to discourage any attempt by users to wait until time 2 to adopt. By substituting the optimal value of control variables in the profit, we obtain: 


$$
\pi^{A^{*}}=2 m\left(b+q^{e}-\bar{b}\right)-\underline{C}
$$

In the case of Strategy A, we can fully specify Assumption 3: :

$$
\underline{c}>2\left(b+q^{e}-\bar{b}\right)
$$

Strategy B: The publisher here determines the level of its control variables such that all potential users that chose the reservation strategy at time 1, finally buy the software at time 2. Given Lemmas 2 and 5, there is no possibility for having simultaneously some users who buy the software at time 1 and others who intend to buy it at time 2. Given Lemma 4, software adoption cannot be postponed by any motive other than a positive revision to the expected software quality resulting from the diffusion of favourable information disclosed by early unauthorized users. Given Lemmas 4 and 5, this permissive strategy then is compatible with the distribution of users defined by Outcome (2. Consequently, Strategy B is optimal for the publisher if the information disclosed by early unauthorized users is favourable to the editor and sufficiently large. At time 1, the publisher must then choose a price $p_{1}$ and a future control level $\lambda_{2}^{a}=1$ sufficiently high to deter potential users from purchase at time 1 or from unauthorized adoption at time 2 . This choice leads to the following conditions $(i \in[0, m])$ :

$$
\left\{\begin{aligned}
1.5 \succ^{i} 1.1 & \Leftrightarrow p_{1}>2\left(q^{e}-\bar{b}\right) \\
1.5 \succ 1.3 & \Leftrightarrow p_{2}^{a}>b+q^{e}-\bar{b} \\
1.5 \succ 1.4 & \Leftrightarrow \lambda_{2}^{a} \underline{c}>q^{e}-\bar{b}
\end{aligned}\right.
$$

The last inequality is always verified if $\lambda_{2}^{a}=1$. It is indeed sufficient for the publisher to announce a maximal level of control at time 2 (which it will automatically do given Lemma 5) in order to ensure that Strategy 1.5 is preferred to Strategy 1.4 given Assumption 3. At the same time, the publisher must select a monitoring level sufficiently permissive to motivate some users to get unauthorized copies at time 1. Consequently, potential users' choices are such that some (but not all) of them prefer to adopt through piracy at time 1 instead of waiting for some new information and, if relevant, potentially adopting later. Two conditions are then associated to these choices:

$$
\underline{c} \lambda_{1}<2\left(q^{e}-\bar{b}\right) \leq \bar{c} \lambda_{1}
$$

When these two conditions are verified, potential users distribute in each time period and for the two time periods according to the outcome defined by Outcome 2 , and the piracy cost of the "marginal unauthorized user" at time 1 is $c_{i}^{*}=\frac{2\left(q^{e}-\bar{b}\right)}{\lambda_{1}}$. At time 2 , the potential users who have not yet adopted, all buy. For all users $i$ whose piracy cost is between $c_{i}^{*}$ et $\bar{c}$, the following conditions then hold:

$$
\begin{cases}2.1 \succeq 2.2 & \Leftrightarrow p_{2} \leq \lambda_{2} \underline{\underline{c}} \\ 2.1 \succeq 2.3 & \Leftrightarrow p_{2} \leq q^{e}-\bar{b}+\frac{k\left(q-q^{e}\right)\left(\frac{2\left(q^{e}-\bar{b}\right)}{\lambda_{1}}-\underline{c}\right)}{\bar{c}-\underline{c}}\end{cases}
$$

The profit associated with Strategy B can be expressed as: $\pi^{B}\left(p_{2}, \lambda_{1}, q\right)=\frac{\bar{c}-\frac{2\left(q^{e}-\bar{b}\right)}{\lambda_{1}}}{\bar{c}-\underline{c}} m p_{2}-$ $C(q)$. 
Given the linearity of the profit function with respect to $p_{2}$, the definition set of $p_{2}$ is compact. Because of Assumption 3, the first inequality $\left(p_{2} \leq \lambda_{2}\right)$ is always checked: as a consequence, the value of $p_{2}$ which maximizes the profit $\pi^{B}\left(p_{2}, \lambda_{1}, q\right)$ is defined by the upper bound of the second inequality, i.e. $p_{2}=q^{e}-\bar{b}+\frac{k\left(q-q^{e}\right)\left(\frac{2\left(q^{e}-\bar{b}\right)}{\lambda_{1}}-\underline{c}\right)}{\bar{c}-\underline{c}}$. This value of $p_{2}$ then can be substituted into the profit function. The costs properties define a corner solution value $q^{*}=\bar{q}$ for software quality. Given Lemmas 4 and $5, \lambda_{2}^{*}=\lambda_{2}^{a *}=1$. The optimal solutions $p_{2}^{*}$ et $\lambda_{1}^{*}$ are deduced from the first order conditions and from the boundary values of the control variables:

$$
\left\{\begin{array}{l}
p_{2}^{*}=\frac{1}{2}\left[q^{e}-\bar{b}+k\left(\bar{q}-q^{e}\right)\right] \\
\lambda_{1}^{*}=\frac{4\left(q^{e}-\bar{b}\right)\left(\bar{q}-q^{e}\right) k}{(\bar{c}+\underline{c})\left(\bar{q}-q^{e}\right) k-(\bar{c}-\underline{c})\left(q^{e}-\bar{b}\right)}
\end{array}\right.
$$

¿From the previous conditions, we can deduce the optimal values of the control variables $\left\{p_{1}, p_{2}^{a}, \lambda_{2}, \lambda_{2}^{a}\right\}$ which only intervene indirectly in the profit expression: $\left\{p_{1}^{*}>\right.$ $2\left(q^{e}-\bar{b}\right), p_{2}^{a *}=p_{2}^{*}=1 / 2\left(q^{e}-\bar{b}+k\left(\bar{q}-q^{e}\right), \lambda_{2}^{*}=\lambda_{2}^{a *}=1\right\}$

Note however that the optimal level of control $\lambda_{1}{ }^{*}$ is greater than zero as soon as the following inequality is satisfied:

$$
k \geq \frac{q^{e}-\bar{b}}{\bar{q}-q^{e}}
$$

In other words, if, for a given level of initial expectations about software quality, the magnitude of information diffusion about software quality is not sufficiently good between the two periods, then the publisher will have to choose a monitoring level equal to zero: all potential users should then choose piracy, which is incompatible with Strategy B. When Condition (4) is satisfied, this case is excluded. Thus, this condition, which states that the level of initial expectations is not too low compared to the potential diffusion of information between the two periods, is a necessary condition for Strategy B to be used by the publisher.

It is important to note that if the condition defined by Equation (4) holds, and if the inequalities associated with Assumption 3 are checked, then $\lambda_{1}^{*}$ is always smaller than 1 . In other words, if it can be shown that for some parameter values, Strategy B is optimal for the publisher, it can be shown also that the publisher has an incentive to adopt a permissive strategy regarding piracy.

After substituting the optimal values of the control variables in the profit, we obtains:

$$
\pi^{B^{*}}=\frac{m\left(q^{e}-\bar{b}+k\left(\bar{q}-q^{e}\right)\right)^{2}}{4 k\left(\bar{q}-q^{e}\right)}-\bar{C}
$$

In the case of Strategy B, Assumption 3 can be fully specified:

$$
\underline{c}>\frac{1}{2}\left(\left(q^{e}-\bar{b}\right)+\left(\bar{q}-q^{e}\right) k\right)
$$

Finally, we need to determine whether Strategy B might be a dominant strategy for the publisher. To do so, we compare the optimal profits associated with Strategies A and $\mathrm{B}\left(\pi^{A^{*}}\right.$ and $\pi^{B^{*}}$ respectively) from which Proposition 1 is deduced: 
Proposition 1. Under certain conditions, a permissive attitude towards piracy during the initial adoption stage $\left(\lambda_{1}<1\right)$ is optimal for the software publisher.

Proof. Let us consider the situation where Strategies A and B are simultaneously possible (i.e. parameter configuration such that Condition (4) holds). In this situation, $\pi^{B^{*}}>\pi^{A^{*}}$ as soon as the information diffusion on software quality is sufficiently high and the provision cost of advanced functionalities is sufficiently low. Formally, Strategy B is optimal if (i) $k>(3+2 \sqrt{2}) \frac{q^{e}-\bar{b}}{\bar{q}-q^{e}}$ and (ii) $\bar{C}<\underline{C}+\frac{m\left(\bar{b}^{2}+6 \bar{b} k \bar{q}+k^{2} \bar{q}^{2}+2 q^{e}(-\bar{b}(1+3 k)-k(3+k) q)+q^{e 2}\left(1+6 k+k^{2}\right)\right)}{4 k\left(\bar{q}-q^{e}\right)}$

Proposition 1 states that implementing a policy that would deter all unauthorized behaviour (i.e. $\lambda=1$ ) is not always profitable. Even if deterring piracy is at no direct cost, the software publisher might have an incentive to tolerate unauthorize behaviours during the early adoption stage of its software. However, this should apply only if these unauthorized behaviours succeed in providing incentives for other potential users to buy the software later. A permissive policy towards piracy then is profitable under two kinds of conditions. First, the publisher must propose a software of a sufficiently high quality level (i.e. measured here by the amount of advanced functionalities provided to users), since unauthorized users subsequently will contribute to the disclosure of this quality level to potential buyers. On the contrary, if the software does not provide a sufficiently high increase in utility to users (compared to some older-generation software as defined by the reservation utility), the nature of the information disclosed by unauthorized users should discourage potential buyers from later adoption. Second, we argue that the anti-piracy policy should solve a trade-off between two elements. On the one hand, it should not be so severe as to allow a large mass of unauthorized users to reveal favourable information about quality or the magnitude of the externality from unauthorized to future legal users will not be sufficient to induce future legal purchase. On the other hand, it should not be so permissive as to induce too many potential users to get unauthorized copies. The software publisher must use monitoring devices that allow it to adjust accurately for the adequate number / proportion of unauthorized users. Proposition 1 states that implementing a policy that would deter all unauthorized behaviour (i.e. $\lambda=1$ ) is not always profitable. Even if deterring piracy is at no direct cost, the software publisher might have an incentive to tolerate unauthorize behaviours during the early adoption stage of its software. However, this should apply only if these unauthorized behaviours succeed in providing incentives for other potential users to buy the software later. A permissive policy towards piracy then is profitable under two kinds of conditions. First, the publisher must propose a software of a sufficiently high quality level (i.e. measured here by the amount of advanced functionalities provided to users), since unauthorized users subsequently will contribute to the disclosure of this quality level to potential buyers. On the contrary, if the software does not provide a sufficiently high increase in utility to users (compared to some older-generation software as defined by the reservation utility), the nature of the information disclosed by unauthorized users should discourage potential buyers from later adoption. Second, we argue that the anti-piracy policy should solve a trade-off between two elements. On the one hand, it should not be so severe as to allow a large mass of unauthorized users to reveal favourable information about quality or the magnitude of the externality from unauthorized to future legal users will not be sufficient to induce future legal purchase. On the other hand, it should not be so permissive as to induce too many potential users to get unauthorized copies. The software publisher must use monitoring devices that allow it to adjust accurately for the adequate number 
/ proportion of unauthorized users.

\subsubsection{Publisher's strategy with misleading announcements}

Now suppose that the software publisher is able and willing to disclose misleading information on the future levels of price and monitoring. Strategies A and B may still be used, but a new strategy (Strategy C) is now available. It consists in announcing a later permissiveness towards piracy. However, this later permissiveness will not be implemented and a non-accommodating strategy towards piracy is be implemented instead.

Strategy C: the publisher chooses the levels of the control variables such that some users immediately buy the software while others plan to obtain it later through piracy. Legal buyers disclose positive information that induces later adoption by other users. However, those users finally are induced to buy the software since the level of monitoring is increased to its maximum level. This strategy is thus based on a misleading announcement about the future permissiveness towards piracy and matches the distribution of users in Outcome 3.

At time 1, the publisher should ensure that some users buy the software. This requires the price $p_{1}$ to be relatively low and the level of monitoring $\lambda_{1}$ maximum. The following three conditions then hold:

$$
\begin{cases}1.1 \succeq 1.2 & \Leftrightarrow p_{1} \leq \lambda_{1} \underline{c} \\ 1.1 \succeq 1.3 & \Leftrightarrow p_{1} \leq p_{2}^{a}+\left(b+q^{e}-\bar{b}\right) \\ 1.1 \succeq 1.5 & \Leftrightarrow p_{1} \leq 2\left(q^{e}-\bar{b}\right)\end{cases}
$$

At the same time, the publisher has to pretend that it will be easier to get unauthorized copies of the software at time 2. Those potential users who are the most able to get unauthorized copies of the software then will believe that getting unauthorized copies of it later (at time 2) is better than buying it immediately (at time 1). For that reason, the current price must be quite high and the future monitoring level particularly low, to provide an incentive for this delayed adoption by users. We then have to check the following double inequality:

$$
\underline{c}<\frac{p_{1}-\left(q^{e}-\bar{b}\right)}{\lambda_{2}^{a}}<\bar{c}
$$

If these two conditions are satisfied, the potential users' choices lead them to be distributed on the segment $[\underline{c}, \bar{c}]$ given by Outcome (3. The potential user $i$ who exhibits the piracy cost $c_{i}^{*}=\frac{p_{1}-\left(q^{e}-\bar{b}\right)}{\lambda_{2}^{a}}$ is indifferent between buying and reservation at time 1 . Note that we consider strict inequalities, i.e. the piracy cost if the indifferent potential user $\left(c_{i}^{*}\right)$ is strictly greater than $\underline{c}$ and strictly less than $\bar{c}$. These strict inequalities are necessary to ensure that potential users distribute among Strategy 1.1 (buying at time 1) and Strategy 1.4 (reservation at time 1 then piracy at time 2). Otherwise, the distribution of potential users defined by Outcome 3 would no longer hold and neither would do Strategy C.

At time 2, the publisher has to ensure that those users who have not adopted at time 1 will buy the software at time 2 . Then the control variables are such that all potential 
users who have initially chosen Strategy 1.4 finally switch to Strategy 2.1:

$$
\begin{cases}2.1 \succeq 2.2 & \Leftrightarrow p_{2} \leq \lambda_{2} \underline{c} \\ 2.1 \succeq 2.3 & \Leftrightarrow p_{2} \leq q^{e}-\bar{b}+\frac{k\left(q-q^{e}\right)\left(\bar{c}-\frac{p_{1}-\left(q^{e}-\bar{b}\right)}{\lambda_{2}^{a}}\right)}{\bar{c}-\underline{c}}\end{cases}
$$

The profit associated with Strategy C is:

$$
\pi^{C}\left(p_{1}, \lambda_{1}, p_{2}, \lambda_{2}^{a}, q\right)=\frac{\bar{c}-\frac{p_{1}-\left(q^{e}-\bar{b}\right)}{\lambda_{2}^{a}}}{\bar{c}-\underline{c}} m p_{1}+\frac{\frac{p_{1}-\left(q^{e}-\bar{b}\right)}{\lambda_{2}^{a}}-\underline{c}}{\bar{c}-\underline{c}} m p_{2}-C(q)
$$

Again (cf. Strategy B), the profit is linear with respect to $p_{2}$. We thus need to bind the more restrictive constraint on $p_{2}$. Because of Assumption 3 , the condition $p_{2} \leq \lambda_{2} \underline{c}$ is always fulfilled and never saturated. Then, $p_{2}^{*}=q^{e}-\bar{b}+\frac{k\left(q-q^{e}\right)\left(\bar{c}-\frac{p_{1}-\left(q^{e}-\bar{b}\right)}{\lambda_{2}^{a}}\right)}{\bar{c}-\underline{c}}$.

Given the set of definition of the control variables, we have:

$$
\left\{\begin{array}{l}
q^{*}=\bar{q} \\
p_{1}^{*}=2\left(q^{e}-\bar{b}\right) \\
p_{2}^{*}=\frac{1}{2}\left[3\left(q^{e}-\bar{b}\right)+k\left(\bar{q}-q^{e}\right)\right] \\
\lambda_{2}^{a *}=\frac{2\left(q^{e}-\bar{b}\right)\left(\bar{q}-q^{e}\right) k}{(\bar{c}+\underline{c})\left(\bar{q}-q^{e}\right) k-(\bar{c}-\underline{c})\left(q^{e}-\bar{b}\right)}
\end{array}\right.
$$

However, note that the control variables $\left\{p_{2}^{a}, \lambda_{1}, \lambda_{2}\right\}$ that do not directly intervene in the profit function have to match Strategy C, which implies $p_{2}^{a *}=p_{2}^{*}$ and $\lambda_{1}^{*}=\lambda_{2}{ }^{*}=1$.

As for Strategy B, enough information needs to be disclosed to ensure that $\lambda_{2}^{a *}$ is different from 0 so that all potential users do not get unauthorized copies of the software a time 2. We then have:

$$
k \geq \frac{q^{e}-\bar{b}}{\bar{q}-q^{e}}
$$

By replacing the optimal values of the control variables in the profit, we deduce:

$$
\pi^{C^{*}}=m\left(q^{e}-\bar{b}\right)+\frac{m\left(q^{e}+\bar{b}+k\left(\bar{q}-q^{e}\right)\right)^{2}}{4 k\left(\bar{q}-q^{e}\right)}-\bar{C}
$$

Finally, in the case of Strategy C, we can fully specify Assumption 3:

$$
\underline{c}>\frac{1}{2}\left(3\left(q^{e}-\bar{b}\right)+\left(q-q^{e}\right) k\right)
$$

Condition 9 ensures that no potential users choose to get unauthorized copies at time 2 if the publisher chooses $\lambda_{2}=1$ (Strategy 2.2 is then always dominated). Note that this condition is stronger that Condition 6 .

Proposition 2 follows from the comparison of the optimal profits associated with strategies $\mathrm{A}, \mathrm{B}$ and $\mathrm{C}$.

Proposition 2. It could be profitable for the publisher to announce later permissiveness towards piracy and ultimately to deter it. 
Proof. Consider that Condition (4) holds so that the publisher has to choose among Strategies A, B and CWith the optimal profit levels associated with Strategy B and C (expressed by Equations ( 5 and ( 8 respectively), we can write: $\pi^{C^{*}}=m\left(q^{e}-\bar{b}\right)+\pi^{B^{*}}$. It can be seen that Strategy C will always be preferred to Strategy B. Comparison of the profits associated with Strategies A and C (Equations 2 and 8 respectively) shows that Strategy $\mathrm{C}$ will be preferred to Strategy A if the additional revenues derived from Strategy $\mathrm{C}$ are counterbalanced by the additional costs, i.e. if $\frac{m\left(q^{e}+\bar{b}+k\left(\bar{q}-q^{e}\right)\right)^{2}}{4 k\left(\bar{q}-q^{e}\right)}-m\left(q^{e}-\bar{b}\right)>\bar{C}-\underline{C}$

While Proposition 1 highlights the strategic publisher's strategy of tolerance towards piracy, Proposition 2 shows that it could be even more profitable to announce to users future tolerance towards piracy, but not to commit to this announcement. Here, we suppose that the publisher is able to make misleading announcements about its control variables. That is, it is able to suggest a lower monitoring level at time 2 than the one that is finally implemented. Thus, there can be an optimal strategy involving information about software quality being disclosed by "buyer-testers" and not by "unauthorized userstesters". At time 2, those users who planned to get unauthorized copies of the software will ultimately be induced to buy it, not only because of their better perception of the software quality but also because piracy is impossible, since the publisher has chosen a maximum monitoring level.

This strategy is the most profitable for the publisher since (i) all users buy the software and (ii) the price paid by late adopters is finally higher $\left(p_{2}^{C *}>p_{2}^{B *}\right)$. Recall that the more positive the information that is diffused about software quality (which depends on the number of early adopters), the higher will be the utility of potential users at time 2 , and the higher the price of the software at time 2 (because it increases willingness-to-pay). With Strategy B, users who provide information about software quality are unauthorized users whereas with Strategy $\mathrm{C}$ they are buyers. For that reason, the software publisher will do better from restricting the number of "unauthorized user-testers" than restricting the number of "buyer-testers". All things being equal, since more information diffuses with Strategy C than with Strategy B, the price at time 2 will be higher with Strategy C.

Note also that, rather surprisingly, with Strategy C, those who pay the higher price are the users who are the better able to get unauthorized copies (lowest piracy cost). In one sense, this outcome could be interpreted as some indirect intertemporal pricediscriminating mechanism. However, this strategy may raise some moral concerns since the publisher intentionally lies about its future monitoring level to limit the number of buyers at time 1 in order to increase the number of buyers at time 2 . In adopting this kind of strategy, the publisher may also loose credibility in the long run which may have a negative impact on its ability to launch a new software or other kinds of products in a repeated game.

\section{Discussion and conclusion}

Based on an original model, our work has several results. Extending some of the results in Takayama [2009], our first result is to show that a distribution strategy based on piracy and indirect sampling can be profitable. This strategy consists in choosing strategically a permissive attitude towards piracy during some early adoption stage (Stage 1 in our 
model) and, thus, generating a word-of-mouth mechanism that helps to disclose information about the software. This fosters future legal adoption of the software. Later, the software may be sold at a higher price because the disclosed information has increased the willingness to pay for legal buyers. The timing of adoption depends on users' piracy cost. Only those users endowed with lower piracy costs adopt during the pre-adoption stage. Those users endowed with higher costs may wait and purchase legally in a second step. From the software publisher's perspective, we verified the optimality of permissive policies towards piracy in a context where getting unauthorized copies of the software has a cost for potential users and where the monitoring of unauthorized behaviours is costless for publishers.

However, it can be argued that, in practice, the "fight" against piracy could generate several types of costs : R\&D costs associated with the development of new tracking technologies, administrative and legal costs linked to potential infringements of Intellectual Property Rights (IPR). In our model, we deliberately assumed these costs to be zero. Doing so enabled us to distinguish clearly between the benefits/costs associated with better information disclosure through a word-of-mouth mechanism and those linked to a specific type of monitoring technology (that would exhibit ad hoc cost conditions). Our results are thus robust to various cost conditions for the monitoring activity. This assumption is the more unfavourable to obtain this first result which in turn reinforces its robustness. Indeed, considering alternative assumptions about piracy and control costs would simply extend the range of the cases where permissive strategies are optimal.

Second, our setting explicitly integrates the announcement made by the publisher about its present and future price and control policies. In this way, our model confirms and extends some results already identified in the literature on piracy. In order to maximize its profit, the publisher can use not only its price and control policies but also its - sincere or not - announcements about its future price and control policies. The publisher can, for example, announce future levels of control of piracy that it does not intend to apply. We showed that when this strategy and the permissive strategy are both admissible, it is even more interesting to cheat - i.e. to announce a future permissive policy that will not be effective - than to be permissive immediately. In this case, the price of the software for the initial period is chosen sufficiently low to attractive those potential users characterized by the the smaller propensity to download illegally (higher piracy costs). These early adopters buy the software and diffuse favourable information to later adopters. Early adopters transmit favourable information about software quality to potential users who initially intended to acquire it illegally at the second time period. This information increases their propensity to adopt. This is sufficient for the publisher to apply an unexpected severe control policy to convert the "future unauthorized users" into "current buyers" during the second time period.

These two results are based on two types of information asymmetry between the software publisher and the potential users. A first type of asymmetry is information about software quality: the software publisher knows the precise quality of the software while potential users have to learn about it (either directly or indirectly). A second and more subtle type of asymmetry is about the structure of the game and the way users form their expectations. In this paper, we considered that users form basic expectations, meaning 
that they do not fully analyse the strategy of the software publisher and infer from it additional information. Namely, users are able to take the best decisions given the announces of software publisher, but they are not able to imagine that software publisher could adapt its announces to the nature of these decisions. This attitude seems reasonable: despite not sophisticated, users' decisions are rational. Conversely, more sophisticated users may know the complete structure of the game and from that may infer some information from observation of prices and monitoring levels and from the announcement made by the publisher. For instance, assume that first time period adoptions come only from piracy. Given the initial information, potential users can infer that if the publisher is rational, it expects that subsequent diffusion of information by unauthorized users will increase the propensity for remaining users to adopt (and their willingness to pay). From this additional information, they may able better to assess the credibility of the publisher's announcement about future price and control strategies. In a longer version of this paper, we analysed diverse degrees of sophistication about the rationality of the publisher and the potential users. ${ }^{6}$ This analysis reveals that when the sophistication of expectations increases, the "cheating strategy" can no longer be used by the software publisher. The reason is that when the publisher announces a later decrease in the monitoring level, this announcement is no longer credible: since the publisher can control potential unauthorized users without cost, it is never optimal for the publisher to be permissive during the last time period when there is nothing to expect from last adopters in terms of information diffusion. Hence, potential users know that any announcement characterized by a permissive strategy toward piracy during the last adoption stage, is not credible. In contrast, the same increase in the level of sophistication of users and publisher confirms the optimality of the strategy analysed in Proposition 1. In particular, if potential users observe that all adopt at the same time (or understand that prices make this early adoption the optimal choice for all), they might conclude that software quality is low and refuse to buy the software at time 1. Finally, the strategy including a controlled level of piracy during the initial time period is revealed as the most robust when the degree of sophistication of potential users increases.

More generally, these results cast doubt on the legitimacy of a technical or legal device that potentially would exclude any type of piracy (if it were ever possible). As a full piracy cannot be optimal, our model suggests instead more subtle strategies dealing with piracy. Considering the broader debate on the scope and enforcement of IPR, this suggests that some highly protecting legal devices even legitimated by the scope of IPR, may finally not be fully enforced by the holder of these IPR. In the context of software adoption (depending on e.g. the type of software and the amount of uncertainty associated with software adoption), our model suggests that software publishers may decide not to fully enforce these rights in certain contexts.

\section{References}

Belleflamme P. and M. Peitz (2010), "Digital Piracy: Theory", CESifo Working Paper, no. 3222, CESifo Group Munich.

Conner K.R., and R.P. Rumelt (1991), "Software Piracy: An Analysis of Protection

\footnotetext{
${ }^{6}$ The detailed results as well as the different proofs are available on request from the authors.
} 
Strategies", Management Science, February, 37, 2, 125-139.

Darmon E., A. Rufini and D. Torre (2009), "Back to software 'profitable piracy': the role of information diffusion, Economics Bulletin, 29, 2, 543-553.

Duchêne A. and P. Waelbroeck (2005), "Peer-to-peer, piracy and the copyright law: implications for consumers and artists", in Takeyama, L.N., Gordon, W.J., Towse, R. (eds.), Developments in the Economics of Copyright. Edward Elgar, UK and USA, 60-79.

Gopal R., and S. Bhattacharjee (2006), "Do artists benefit from online music sharing?", Journal of Business, 79, 1503-1534.

Haruvy, E., and A. Prasad (2005), "Freeware as a competitive deterrent" , Information Economics and Policy, 17, 4, 513-534.

Liebowitz, S. J. (2006), "Economists Examine File-Sharing and Music Sales", in Illing, G, and Peitz, M. (eds.), The Industrial Organization And The Digital Economy, MIT Press, 145-170.

Nelson, P. (1970), "Information and Consumer Behavior", Journal of Political Economy, 78, 2, 311-329.

Peitz M., and P. Waelbroeck (2006a), "Piracy of digital products: A critical review of the theoretical literature", Information Economics and Policy, 18, 4, 449-476.

Peitz M., and P. Waelbroeck (2006b), "Why the music industry may gain from free downloading - the role of sampling", International Journal of Industrial Organization, 24, 900-913.

Shapiro C., and H.R. Varian (1998), Information Rules: A Strategic Guide to the Network Economy, Harvard Business School Press.

Stolpe M. (2000), "Protection against software piracy: a study of technology adoption for the enforcement of intellectual property rights", Economics of Innovation and New Technology, 9, 25-52.

Takeyama, L. (2009), "Copyright enforcement and product quality signaling in markets for computer software", Information Economics and Policy, 21, 291-296

Varian, H.R. (2005), "Copying and Copyright", Journal of Economic Perspectives, 19, $121-38$ 